\title{
Exploring Prime Numbers and Modular Functions I: On the Exponential of Prime Number via Dedekind Eta Function
}

\author{
Edigles Guedes ${ }^{1}$ and Prof. Dr. K. Raja Rama Gandhi ${ }^{2}$ \\ Number Theorist, Brazil ${ }^{1}$ \\ Resource perosn in Mathematics for Oxford University Press and Professor at BITS-Vizag ${ }^{2}$
}

ABSTRACT. The main objective this paper is to develop asymptotic formulas for the exponential of prime number, using Dedekind eta function, and afterwards an elliptic modular function.

\section{INTRODUCTION}

As consequence of the prime number theorem, I get the asymptotic formula for the $n$th prime number, denoted by $p_{n}$ :

$$
p_{n} \sim n \ln n .
$$

M. Pervouchine, in Mémories de la Société physic-mathématique de Kasan, [1, page 848] deduced that

$$
\frac{p_{n}}{n} \approx \ln n+\ln \ln n-1+\frac{5}{12 \ln n}+\frac{1}{24 \ln \ln n} .
$$

On the other hand, Ernest Cesáro, in Sur une formule empirique de M. Pervouchine [1, page 849], I encounter the formula

$$
\frac{p_{n}}{n} \approx \ln n+\ln \ln n-1+\frac{\ln \ln n-2}{\ln n}-\frac{(\ln \ln n)^{2}-6 \ln \ln n+11}{2(\ln n)^{2}}
$$

in modern notation,

$$
\frac{p_{n}}{n}=\ln n+\ln \ln n-1+\frac{\ln \ln n-2}{\ln n}-\frac{(\ln \ln n)^{2}-6 \ln \ln n+11}{2(\ln n)^{2}}+O\left(\frac{1}{(\ln n)^{2}}\right) .
$$

On the other hand, the Dedekind eta function was introduced by Richard Dedekind, in 1877, and is defined in the half-plane $\mathbb{H}=\{\tau: \mathfrak{J}(\tau)>0\}$ by the equation [2, page 47]

$$
\eta(\tau):=e^{\pi i \tau / 12} \prod_{k=1}^{\infty}\left(1-e^{2 \pi i k \tau}\right)
$$

The infinite product has the form $\Pi\left(1-x^{n}\right)$, where $x=e^{2 \pi i \tau}$. If $\tau \in \mathbb{H}$, then $|x|<1$, so the product converges absolutely and is nonzero.

In [2, page 48], I have

$$
\frac{1}{2} \ln y=\ln \eta(i / y)-\ln \eta(i y)
$$

hence,

$$
\ln y=2[\ln \eta(i / y)-\ln \eta(i y)]=2 \ln \left[\frac{\eta(i / y)}{\eta(i y)}\right]
$$

for $y>0$. 
In this paper, I prove, among other things, that

$$
\begin{gathered}
e^{\frac{p_{n}}{2 n}} \approx \frac{\eta\left\{i /\left[n \ln n e^{\left.\left.\frac{1}{24}\left(\frac{1}{\ln \ln n}+\frac{10}{\ln n}-24\right)\right]\right\}}\right.\right.}{\eta\left[i n \ln n e^{\left.\frac{1}{24}\left(\frac{1}{\ln \ln n}+\frac{10}{\ln n}-24\right)\right]}\right.} \\
e^{\frac{p_{n}}{2 n}-\frac{\pi n}{12}} \sum_{k=-\infty}^{\infty}(-1)^{k} e^{-\pi n k(3 k-1)} \sim e^{-\frac{\pi}{12 n}} \sum_{k=-\infty}^{\infty}(-1)^{k} e^{-\frac{\pi k(3 k-1)}{n}}, \\
e^{\frac{p_{n}}{2 n}} \sum_{k=-\infty}^{\infty}(-1)^{k} e^{-\frac{\pi n(6 k-1)^{2}}{12}} \sim \sum_{k=-\infty}^{\infty}(-1)^{k} e^{-\frac{\pi(6 k-1)^{2}}{12 n}}
\end{gathered}
$$

and

$$
\begin{aligned}
& e^{\frac{p_{n}}{2 n}-\frac{\pi n}{12}}\left\{1+\sum_{k=1}^{\infty}(-1)^{k}\left[e^{-\pi n k(3 k-1)}+e^{-\pi n k(3 k+1)}\right]\right\} \sim e^{-\frac{\pi}{12 n}}\{1 \\
+ & \left.\sum_{k=1}^{\infty}(-1)^{k}\left[e^{-\frac{\pi k(3 k-1)}{n}}+e^{-\frac{\pi k(3 k+1)}{n}}\right]\right\}
\end{aligned}
$$

\section{THEOREMS}

\section{PART 1}

In this part, I develop one asymptotic connection between elliptic modular functions, more specifically, the Dedekind eta function, and the exponential function of a prime number.

THEOREM 1. I have

$$
e^{\frac{p_{n}}{2 n}} \approx \frac{\eta\left\{i /\left[n \ln n e^{\left.\left.\frac{1}{24}\left(\frac{1}{\ln \ln n}+\frac{10}{\ln n}-24\right)\right]\right\}}\right.\right.}{\eta\left[i n \ln n e^{\left.\frac{1}{24}\left(\frac{1}{\ln \ln n}+\frac{10}{\ln n}-24\right)\right]}\right.}
$$

where $p_{n}$ denotes the $n$th prime number and $\eta(\tau)$ denotes the Dedekind eta function.

Proof. By (2), I have

$$
\begin{aligned}
& \frac{p_{n}}{n} \approx \ln n+\ln \ln n-1+\frac{5}{12 \ln n}+\frac{1}{24 \ln \ln n} \\
& =\ln n+\ln \ln n-\ln e+\ln \left(e^{\frac{5}{12 \ln n}}\right)+\ln \left(e^{\frac{1}{24 \ln \ln n}}\right) \\
& =\ln \left(n \ln n e^{\frac{5}{12 \ln n}+\frac{1}{24 \ln \ln n}-1}\right)=\ln \left[n \ln n e^{\frac{1}{24}\left(\frac{1}{\ln \ln n}+\frac{10}{\ln n}-24\right)}\right] \text {. }
\end{aligned}
$$

I substitute (7) in the right hand side of (8), and obtain

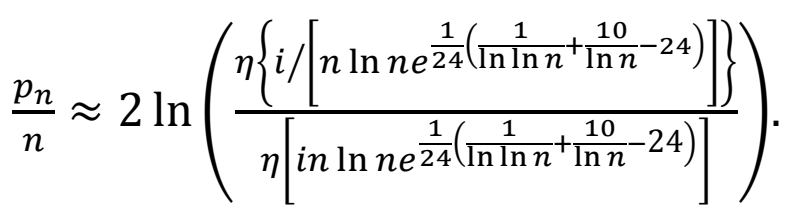


The exponentiation of (9) give me

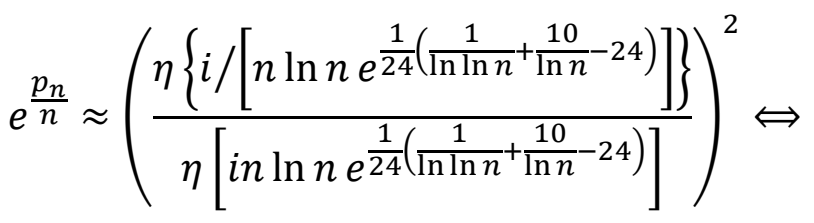

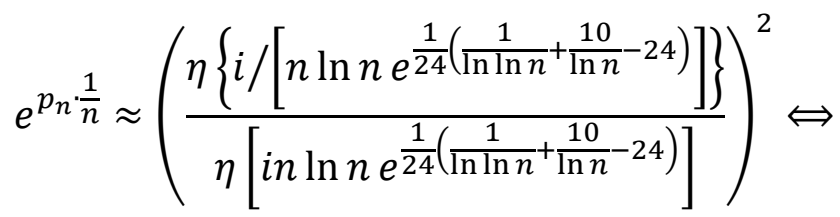

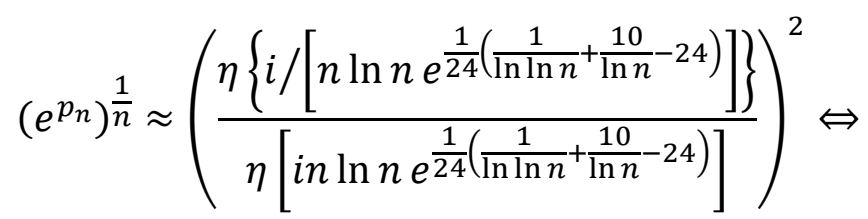

$$
\begin{aligned}
& e^{\frac{p_{n}}{2 n}} \approx \frac{\eta\left\{i /\left[n \ln n e^{\left.\left.\frac{1}{24}\left(\frac{1}{\ln \ln n}+\frac{10}{\ln n}-24\right)\right]\right\}}\right.\right.}{\eta\left[i n \ln n e^{\frac{1}{24}\left(\frac{1}{\ln \ln n}+\frac{10}{\ln n}-24\right)} . \square\right.}
\end{aligned}
$$

COROLLARY 1. I have

$$
e^{\frac{p_{n}}{2 n}} \sim \frac{\eta\left\{i /\left[n\left(10+\frac{\ln n}{\ln \ln n}\right)\right]\right\}}{\eta\left[i n\left(10+\frac{\ln n}{\ln \ln n}\right)\right]}
$$

where $p_{n}$ denotes the $n$th prime number and $\eta(\tau)$ denotes the Dedekind eta function.

Proof. The representation in series power of $e^{\frac{1}{24}\left(\frac{1}{\ln \ln n}+\frac{10}{\ln n}-24\right)}$ is

$$
e^{\frac{1}{24}\left(\frac{1}{\ln \ln n}+\frac{10}{\ln n}-24\right)}=1+\frac{1}{24}\left(\frac{1}{\ln \ln n}+\frac{10}{\ln n}-24\right)+\frac{1}{2 !}\left[\frac{1}{24}\left(\frac{1}{\ln \ln n}+\frac{10}{\ln n}-24\right)\right]^{2}+\cdots,
$$

so,

$$
e^{\frac{1}{24}\left(\frac{1}{\ln \ln n}+\frac{10}{\ln n}-24\right)} \sim 1+\frac{1}{24}\left(\frac{1}{\ln \ln n}+\frac{10}{\ln n}-24\right)=\frac{1}{\ln \ln n}+\frac{10}{\ln n} .
$$

I substitute the above result in Theorem 1, and this completes the proof.

THEOREM 2. I have

$$
e^{\frac{p_{n}}{2 n}} \sim \frac{\eta(i / n)}{\eta(i n)}
$$

where $p_{n}$ denotes the $n$th prime number and $\eta(\tau)$ denotes the Dedekind eta function.

Proof. By (1), I find

$$
\frac{p_{n}}{n} \sim \ln n
$$


I substitute (7) in the right hand side of (10), and obtain

$$
\frac{p_{n}}{n} \sim 2 \ln \left[\frac{\eta(i / n)}{\eta(i n)}\right]
$$

The exponentiation of (11) give me

$$
\begin{gathered}
e^{\frac{p_{n}}{n}} \sim\left[\frac{\eta(i / n)}{\eta(i n)}\right]^{2} \Leftrightarrow \\
e^{p_{n} \cdot \frac{1}{n}} \sim\left[\frac{\eta(i / n)}{\eta(i n)}\right]^{2} \Leftrightarrow \\
\left(e^{\left.p_{n}\right)^{\frac{1}{n}}} \sim\left[\frac{\eta(i / n)}{\eta(i n)}\right]^{2} \Leftrightarrow\right. \\
e^{\frac{p_{n}}{2 n}} \sim \frac{\eta(i / n)}{\eta(i n)} \cdot \square
\end{gathered}
$$

THEOREM 3. I have

$$
e^{\frac{p_{n}}{2 n}} \approx \frac{\eta\left(i /\left\{n \ln n e^{-\frac{1}{2(\ln n)^{2}}\left[(\ln \ln n)^{2}-2(\ln n+3) \ln \ln n+4 \ln n+2(\ln n)^{2}+11\right]}\right\}\right)}{\eta\left\{i n \ln n e^{-\frac{1}{2(\ln n)^{2}}\left[(\ln \ln n)^{2}-2(\ln n+3) \ln \ln n+4 \ln n+2(\ln n)^{2}+11\right]}\right\}}
$$

where $p_{n}$ denotes the $n$th prime number and $\eta(\tau)$ denotes the Dedekind eta function.

Proof. By (2), I meet

$$
\begin{aligned}
& \frac{p_{n}}{n} \approx \ln n+\ln \ln n-1+\frac{\ln \ln n-2}{\ln n}-\frac{(\ln \ln n)^{2}-6 \ln \ln n+11}{2(\ln n)^{2}} \\
& =\ln n+\ln \ln n-\ln e+\ln \left(e^{\frac{\ln \ln n-2}{\ln n}}\right)-\ln \left(e^{\frac{(\ln \ln n)^{2}-6 \ln \ln n+11}{2(\ln n)^{2}}}\right) \\
& =\ln \left(n \ln n e^{\frac{\ln \ln n-2}{\ln n}-\frac{(\ln \ln n)^{2}-6 \ln \ln n+11}{2(\ln n)^{2}}-1}\right) \\
& =\ln \left\{n \ln n e^{\frac{1}{2(\ln n)^{2}}\left[2 \ln n(\ln \ln n-2)-(\ln \ln n)^{2}+6 \ln \ln n-11-2(\ln n)^{2}\right]}\right\} \\
& =\ln \left\{n \ln n e^{-\frac{1}{2(\ln n)^{2}}\left[(\ln \ln n)^{2}-2(\ln n+3) \ln \ln n+4 \ln n+2(\ln n)^{2}+11\right]}\right\} .
\end{aligned}
$$

I take (12) in (7), and obtain

$$
\frac{p_{n}}{n} \approx 2 \ln \left[\frac{\eta\left(i /\left\{n \ln n e^{-\frac{1}{2(\ln n)^{2}}\left[(\ln \ln n)^{2}-2(\ln n+3) \ln \ln n+4 \ln n+2(\ln n)^{2}+11\right]}\right\}\right)}{\eta\left\{i n \ln n e^{-\frac{1}{2(\ln n)^{2}}\left[(\ln \ln n)^{2}-2(\ln n+3) \ln \ln n+4 \ln n+2(\ln n)^{2}+11\right]}\right\}}\right] .
$$


The exponentiation of (13) give me

$$
\begin{gathered}
e^{\frac{p_{n}}{n}} \approx\left[\frac{\eta\left(i /\left\{n \ln n e^{-\frac{1}{2(\ln n)^{2}}\left[(\ln \ln n)^{2}-2(\ln n+3) \ln \ln n+4 \ln n+2(\ln n)^{2}+11\right]}\right\}\right)}{\eta\left\{i n \ln n e^{-\frac{1}{2(\ln n)^{2}}\left[(\ln \ln n)^{2}-2(\ln n+3) \ln \ln n+4 \ln n+2(\ln n)^{2}+11\right]}\right\}}\right] \\
e^{\frac{p_{n}}{2 n}} \approx \frac{\eta\left(i /\left\{n \ln n e^{-\frac{1}{2(\ln n)^{2}}\left[(\ln \ln n)^{2}-2(\ln n+3) \ln \ln n+4 \ln n+2(\ln n)^{2}+11\right]}\right\}\right)}{\eta\left\{i n \ln n e^{-\frac{1}{2(\ln n)^{2}}\left[(\ln \ln n)^{2}-2(\ln n+3) \ln \ln n+4 \ln n+2(\ln n)^{2}+11\right]}\right\}} .
\end{gathered}
$$

COROLLARY 2. I have

$$
e^{\frac{p_{n}}{2 n}} \sim \frac{\eta\left(-2 i \ln n /\left\{n\left[(\ln \ln n)^{2}-2(\ln n+3) \ln \ln n+4 \ln n+11\right]\right\}\right)}{\eta\left(-\frac{i n}{2 \ln n}\left[(\ln \ln n)^{2}-2(\ln n+3) \ln \ln n+4 \ln n+11\right]\right)},
$$

where $p_{n}$ denotes the $n$th prime number and $\eta(\tau)$ denotes the Dedekind eta function.

Proof. The representation in series power of $e^{-\frac{1}{2(\ln n)^{2}}\left[(\ln \ln n)^{2}-2(\ln n+3) \ln \ln n+4 \ln n+2(\ln n)^{2}+11\right]}$ is

$$
\begin{aligned}
e^{-\frac{1}{2(\ln n)^{2}}\left[(\ln \ln n)^{2}-2(\ln n+3) \ln \ln n+4 \ln n+2(\ln n)^{2}+11\right]} \\
=1-\frac{1}{2(\ln n)^{2}}\left[(\ln \ln n)^{2}-2(\ln n+3) \ln \ln n+4 \ln n+2(\ln n)^{2}+11\right] \\
+\frac{1}{2 !}\left\{\frac{1}{2(\ln n)^{2}}\left[(\ln \ln n)^{2}-2(\ln n+3) \ln \ln n+4 \ln n+2(\ln n)^{2}+11\right]\right\}^{2}+\cdots
\end{aligned}
$$

so,

$e^{-\frac{1}{2(\ln n)^{2}}\left[(\ln \ln n)^{2}-2(\ln n+3) \ln \ln n+4 \ln n+2(\ln n)^{2}+11\right]}$

$$
\sim 1-\frac{1}{2(\ln n)^{2}}\left[(\ln \ln n)^{2}-2(\ln n+3) \ln \ln n+4 \ln n+2(\ln n)^{2}+11\right] .
$$

I substitute the previous result in Theorem 3, and this completes the proof.

THEOREM 4. I have

$$
\begin{array}{r}
e^{\frac{p_{n}}{2 n}-\frac{\pi n}{12}} \sum_{k=-\infty}^{\infty}(-1)^{k} e^{-\pi n k(3 k-1)} \sim e^{-\frac{\pi}{12 n}} \sum_{k=-\infty}^{\infty}(-1)^{k} e^{-\frac{\pi k(3 k-1)}{n}}, \\
e^{\frac{p_{n}}{2 n}} \sum_{k=-\infty}^{\infty}(-1)^{k} e^{-\frac{\pi n(6 k-1)^{2}}{12}} \sim \sum_{k=-\infty}^{\infty}(-1)^{k} e^{-\frac{\pi(6 k-1)^{2}}{12 n}}
\end{array}
$$

and

$$
\begin{aligned}
& e^{\frac{p_{n}}{2 n}-\frac{\pi n}{12}}\left\{1+\sum_{k=1}^{\infty}(-1)^{k}\left[e^{-\pi n k(3 k-1)}+e^{-\pi n k(3 k+1)}\right]\right\} \sim e^{-\frac{\pi}{12 n}}\{1 \\
+ & \left.\sum_{k=1}^{\infty}(-1)^{k}\left[e^{-\frac{\pi k(3 k-1)}{n}}+e^{-\frac{\pi k(3 k+1)}{n}}\right]\right\}
\end{aligned}
$$

where $p_{n}$ denotes the $n$th prime number. 
Proof. In [3], the Dedekind eta function have the following series sum representation

$$
\begin{gathered}
\eta(\tau)=e^{\frac{\pi i \tau}{12}} \sum_{k=-\infty}^{\infty}(-1)^{k} e^{\pi i \tau k(3 k-1)} \\
=\sum_{k=-\infty}^{\infty}(-1)^{k} e^{\frac{\pi i \tau(6 k-1)^{2}}{12}} \\
=e^{\frac{\pi i \tau}{12}}\left\{1+\sum_{k=1}^{\infty}(-1)^{k}\left[e^{\pi i \tau k(3 k-1)}+e^{\pi i \tau k(3 k+1)}\right]\right\}
\end{gathered}
$$

Substituting (14), (15) and (16) in Theorem 2, I obtain

$$
\begin{aligned}
& e^{\frac{p_{n}}{2 n}} \eta(i n) \sim \eta(i / n), \\
& e^{\frac{p_{n}}{2 n}-\frac{\pi n}{12}} \sum_{k=-\infty}^{\infty}(-1)^{k} e^{-\pi n k(3 k-1)} \sim e^{-\frac{\pi}{12 n}} \sum_{k=-\infty}^{\infty}(-1)^{k} e^{-\frac{\pi k(3 k-1)}{n}}, \\
& e^{\frac{p_{n}}{2 n}} \sum_{k=-\infty}^{\infty}(-1)^{k} e^{-\frac{\pi n(6 k-1)^{2}}{12}} \sim \sum_{k=-\infty}^{\infty}(-1)^{k} e^{-\frac{\pi(6 k-1)^{2}}{12 n}}, \\
& e^{\frac{p_{n}}{2 n}-\frac{\pi n}{12}}\left\{1+\sum_{k=1}^{\infty}(-1)^{k}\left[e^{-\pi n k(3 k-1)}+e^{-\pi n k(3 k+1)}\right]\right\} \sim e^{-\frac{\pi}{12 n}}\{1 \\
& \left.+\sum_{k=1}^{\infty}(-1)^{k}\left[e^{-\frac{\pi k(3 k-1)}{n}}+e^{-\frac{\pi k(3 k+1)}{n}}\right]\right\} \text {. }
\end{aligned}
$$

This completes the proof.

\section{PART 2}

In this part, I extend the previous work with the elliptical modular functions, demonstrating other asymptotic formula for the exponential function of prime number.

THEOREM 5. I have

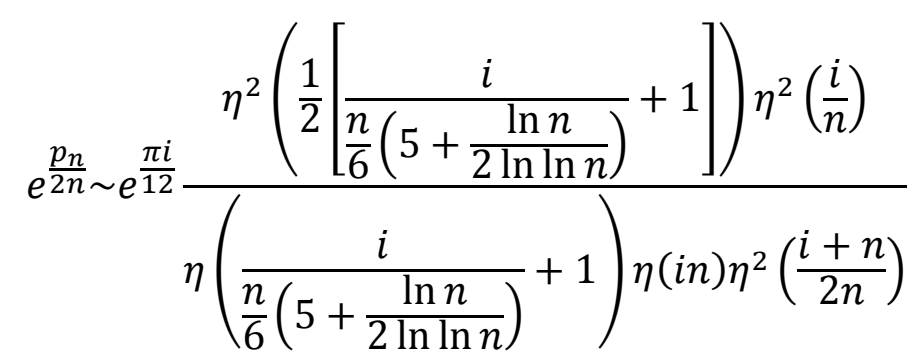

and

$$
e^{\frac{p_{n}}{2 n} \sim} \frac{\eta^{2}\left(\frac{1}{2}\left[\frac{i}{\frac{n}{6}\left(5+\frac{\ln n}{2 \ln \ln n}\right)}+1\right]\right) \eta^{2}\left(\frac{i}{n}\right)}{2 \eta\left(\frac{i}{\frac{n}{6}\left(5+\frac{\ln n}{2 \ln \ln n}\right)}+1\right) \eta(\text { in }) \eta^{2}\left(\frac{2 i}{n}\right)}
$$

where $p_{n}$ denotes the $n$th prime number and $\eta(\tau)$ denotes the Dedekind eta function. 
Proof. In [4, page 114], Weber defined the following functions

$$
\begin{aligned}
& f(\tau)=\frac{e^{-\frac{\pi i}{24} \eta\left(\frac{\tau+1}{2}\right)}}{\eta(\tau)} \\
& f_{2}(\tau)=\sqrt{2} \frac{\eta(2 \tau)}{\eta(\tau)}
\end{aligned}
$$

and conclude that

$$
\begin{gathered}
\vartheta_{00}=\eta(\tau) f(\tau)^{2}, \\
\vartheta_{10}=\eta(\tau) f_{2}(\tau)^{2},
\end{gathered}
$$

where $\vartheta_{00}, \vartheta_{01}$ and $\vartheta_{10}$ are Jacobi theta functions.

The Theorem 2 assures me that

$$
e^{\frac{p_{n}}{2 n}} \eta(i n) \sim \eta(i / n),
$$

Multiplying both members of $(21)$ by $f(i / n)^{2}$ and $f_{2}(i / n)^{2}$, respectively, I encounter

$$
\begin{gathered}
e^{\frac{p_{n}}{2 n}} \eta(\text { in }) f(i / n)^{2} \sim \eta(i / n) f(i / n)^{2}, \\
e^{\frac{p_{n}}{2 n}} \eta(i n) f_{2}(i / n)^{2} \sim \eta(i / n) f_{2}(i / n)^{2},
\end{gathered}
$$

from (17) and (18), I set

$$
\begin{gathered}
e^{\frac{p_{n}}{2 n}} \frac{e^{-\frac{\pi i}{12} \eta(i n) \eta^{2}\left(\frac{i+n}{2 n}\right)}}{\eta^{2}\left(\frac{i}{n}\right)} \sim \vartheta_{00}, \\
2 e^{\frac{p_{n}}{2 n} \frac{\eta(i n) \eta^{2}\left(\frac{2 i}{n}\right)}{\eta^{2}\left(\frac{i}{n}\right)} \sim \vartheta_{10} .}
\end{gathered}
$$

In [5, page 173] the Ramanujan's theta function $f(a, b)$ is defined by

$$
f(a, b):=\sum_{k=-\infty}^{\infty} a^{k(k+1) / 2} b^{k(k-1) / 2}, \quad|a b|<1,
$$

which is a generalization of Jacobi theta functions. Roughly speaking, I can suppose that

$$
\begin{gathered}
e^{\frac{p_{n}}{2 n}} \frac{e^{-\frac{\pi i}{12} \eta(i n) \eta^{2}\left(\frac{i+n}{2 n}\right)}}{\eta^{2}\left(\frac{i}{n}\right)}=f_{00}(a, b), \\
2 e^{\frac{p_{n}}{2 n}} \frac{\eta(i n) \eta^{2}\left(\frac{2 i}{n}\right)}{\eta^{2}\left(\frac{i}{n}\right)}=f_{10}(a, b) .
\end{gathered}
$$

videlicet,

$$
e^{\frac{p_{n}}{2 n}} \frac{e^{-\frac{\pi i}{12}} \eta(i n) \eta^{2}\left(\frac{i+n}{2 n}\right)}{\eta^{2}\left(\frac{i}{n}\right)} \approx \sum_{k=-\infty}^{\infty} e^{-\pi \sigma(n) k(k+1) / 2} e^{-\pi \sigma(n) k(k-1) / 2}=\vartheta_{3}\left(0, e^{-2 \pi \sigma(n)}\right),
$$




$$
2 e^{\frac{p_{n}}{2 n}} \frac{\eta(i n) \eta^{2}\left(\frac{2 i}{n}\right)}{\eta^{2}\left(\frac{i}{n}\right)} \approx \sum_{k=-\infty}^{\infty} e^{-\pi \omega(n) k(k+1) / 2} e^{-\pi \omega(n) k(k-1) / 2}=\vartheta_{3}\left(0, e^{-2 \pi \omega(n)}\right) .
$$

How calculate the functions $\sigma(n)$ and $\omega(n)$ ? Approximately, I have

$$
\begin{aligned}
& e^{\frac{p_{n}}{2 n}} \frac{e^{-\frac{\pi i}{12} \eta(i n) \eta^{2}\left(\frac{i+n}{2 n}\right)}}{\eta^{2}\left(\frac{i}{n}\right)} \approx \\
& \sum_{k=-\infty}^{\infty} e^{-\pi \sigma(n) k(k+1) / 2} e^{-\pi \sigma(n) k(k-1) / 2} \sim \int_{-\infty}^{\infty} e^{-\pi \sigma(n) x(x+1) / 2} e^{-\pi \sigma(n) x(x-1) / 2} d x=\frac{1}{\sqrt{2 \sigma(n)}},
\end{aligned}
$$

$2 e^{\frac{p_{n}}{2 n}} \frac{\eta(i n) \eta^{2}\left(\frac{2 i}{n}\right)}{\eta^{2}\left(\frac{i}{n}\right)} \approx$

$\sum_{k=-\infty}^{\infty} e^{-\pi \omega(n) k(k+1) / 2} e^{-\pi \omega(n) k(k-1) / 2} \sim \int_{-\infty}^{\infty} e^{-\pi \omega(n) x(x+1) / 2} e^{-\pi \omega(n) x(x-1) / 2} d x=\frac{1}{\sqrt{2 \omega(n)}}$,

pursuant to,

$$
\begin{gathered}
\sigma(n) \sim \frac{\eta^{4}\left(\frac{i}{n}\right)}{2 e^{\frac{p_{n}}{n} e^{-\frac{\pi i}{6}} \eta^{2}(i n) \eta^{4}\left(\frac{i+n}{2 n}\right)^{2}}} \\
\omega(n) \sim \frac{\eta^{4}\left(\frac{i}{n}\right)}{8 e^{\frac{p_{n}}{n}} \eta^{2}(i n) \eta^{4}\left(\frac{2 i}{n}\right)}
\end{gathered}
$$

From (8), (33) and (34), I obtain

$$
\begin{gathered}
\sigma(n) \sim \frac{e^{\frac{\pi i}{6}} \eta^{4}\left(\frac{i}{n}\right)}{2 n \ln n e^{\frac{1}{12}\left(\frac{5}{\ln n}+\frac{1}{2 \ln \ln n}-12\right)} \eta^{2}(i n) \eta^{4}\left(\frac{i+n}{2 n}\right)^{2}}, \\
\omega(n) \sim \frac{\eta^{4}\left(\frac{i}{n}\right)}{8 n \ln n e^{\frac{1}{12}\left(\frac{5}{\ln n}+\frac{1}{\left.2 \ln \ln n^{-12}\right)} \eta^{2}(i n) \eta^{4}\left(\frac{2 i}{n}\right)\right.}} .
\end{gathered}
$$

On the other hand, I notice that

$$
\begin{gathered}
\frac{\eta^{4}\left(\frac{i}{n}\right)}{\eta^{2}(i n) \eta^{4}\left(\frac{i+n}{2 n}\right)} \searrow \frac{\sqrt{3}}{2}-\frac{1}{2} i, \\
\frac{\eta^{4}\left(\frac{i}{n}\right)}{\eta^{2}(i n) \eta^{4}\left(\frac{2 i}{n}\right)} \searrow 4 .
\end{gathered}
$$

I get (37) and (38) into (35) and (36)

$$
\begin{aligned}
& \sigma(n) \sim \frac{e^{\frac{\pi i}{6}\left(\frac{\sqrt{3}}{2}-\frac{1}{2} i\right)}}{2 n \ln n e^{\frac{1}{12}\left(\frac{5}{\ln n}+\frac{1}{2 \ln \ln n}-12\right)}} \\
& \omega(n) \sim \frac{1}{2 n \ln n e^{\frac{1}{12}\left(\frac{5}{\ln n}+\frac{1}{2 \ln \ln n}-12\right)}} .
\end{aligned}
$$


On the one hand, I readily saw that

$$
e^{\frac{\pi i}{6}}=\frac{\sqrt{3}}{2}+\frac{1}{2} i
$$

and the representation in series power of $e^{\frac{1}{24}\left(\frac{1}{\ln \ln n}+\frac{10}{\ln n}-24\right)}$ is

$$
e^{\frac{1}{24}\left(\frac{1}{\ln \ln n}+\frac{10}{\ln n}-24\right)}=1+\frac{1}{24}\left(\frac{1}{\ln \ln n}+\frac{10}{\ln n}-24\right)+\frac{1}{2 !}\left[\frac{1}{24}\left(\frac{1}{\ln \ln n}+\frac{10}{\ln n}-24\right)\right]^{2}+\cdots
$$

I set (41) and (42) in (39) and (40)

$$
\begin{aligned}
& \sigma(n) \sim \frac{1}{2 n \ln n\left[1+\frac{1}{12}\left(\frac{5}{\ln n}+\frac{1}{2 \ln \ln n}-12\right)\right]}=\frac{1}{n\left[\frac{1}{6}\left(5+\frac{\ln n}{2 \ln \ln n}\right)\right]} \\
& \omega(n) \sim \frac{1}{2 n \ln n\left[1+\frac{1}{12}\left(\frac{5}{\ln n}+\frac{1}{2 \ln \ln n}-12\right)\right]}=\frac{1}{n\left[\frac{1}{6}\left(5+\frac{\ln n}{2 \ln \ln n}\right)\right]}
\end{aligned}
$$

From (29), (30), (43) and (44), I conclude that

$$
\begin{aligned}
& e^{\frac{p_{n}}{2 n}} \sim \frac{e^{\frac{\pi i}{12}} \vartheta_{3}\left(0, e^{-\frac{\pi}{\frac{n}{6}\left(5+\frac{\ln n}{2 \ln \ln n}\right)}}\right) \eta^{2}\left(\frac{i}{n}\right)}{\eta(i n) \eta^{2}\left(\frac{i+n}{2 n}\right)}, \\
& e^{\frac{p_{n}}{2 n}} \sim \frac{\vartheta_{3}\left(0, e^{\left.-\frac{n}{\frac{n}{6}\left(5+\frac{\ln n}{2 \ln \ln n}\right)}\right)}\right) \eta^{2}\left(\frac{i}{n}\right)}{2 \eta(i n) \eta^{2}\left(\frac{2 i}{n}\right)} .
\end{aligned}
$$

In [3], I encounter

$$
\vartheta_{3}\left(0, e^{\pi i \tau}\right)=\frac{\eta^{2}\left(\frac{\tau+1}{2}\right)}{\eta(\tau+1)} .
$$

I substitute (47) into (45) and (46)

$$
\begin{gathered}
e^{\frac{p_{n}}{2 n} \sim e^{\frac{\pi i}{12}}} \frac{\eta^{2}\left(\frac{1}{2}\left[\frac{i}{\frac{n}{6}\left(5+\frac{\ln n}{2 \ln \ln n}\right)}+1\right]\right) \eta^{2}\left(\frac{i}{n}\right)}{\eta\left(\frac{i}{\frac{n}{6}\left(5+\frac{\ln n}{2 \ln \ln n}\right)}+1\right) \eta(i n) \eta^{2}\left(\frac{i+n}{2 n}\right)} \\
e^{\frac{p_{n}}{2 n} \sim} \frac{\eta^{2}\left(\frac{1}{2}\left[\frac{i}{\frac{n}{6}\left(5+\frac{\ln n}{2 \ln \ln n}\right)}+1\right]\right) \eta^{2}\left(\frac{i}{n}\right)}{2 \eta\left(\frac{i}{\frac{n}{6}\left(5+\frac{\ln n}{2 \ln \ln n}\right)}+1\right) \eta(i n) \eta^{2}\left(\frac{2 i}{n}\right)} . \square
\end{gathered}
$$




\section{REFERENCES}

[1] Cesàro, Ernest, Sur une formule empirique de M. Pervouchine, Comptes rendus hebdomadaires des séances de l'Academia des sciences 119: 848-849, (1894). (French)

[2] Apostol, Tom M., Modular functions and Dirichlet series in number theory, Springer Verlag, 2000.

[3] Iisstein, Eric W., Dedekind Eta Function, from MathWorld - A Wolfram Ib Resource, http://mathworld.wolfram.com/DedekindEtaFunction.html, available in November 17, 2013.

[4] Iber, Heinrich, Lehrbuch der Algebra, Vol. 3, 1908.

[5] Andrews, George E. and Berndt, Bruce C., Ramanujan's Lost Notebook, Part II, Springer, 2009.

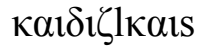

